\title{
¿CÓMO MEDIMOS EL SERVICIO?
}

José Domingo Begazo Villanueva *

E-mail: jbegazov@unmsm.edu.pe

\section{RESUMEN}

En la venta de productos o servicios, el marketing cumple un rol importante al identificar las necesidades y expectativas de los consumidores. Se analiza lo que los clientes requieren al comprar un producto o servicio, sus preferencias, sus satisfacciones y sus requerimientos.

La tangibilidad de un producto va acompañada de la intangibilidad de las expectativas que deben ser medidas en función a las percepciones de los clientes. De eso se trata el presente artículo que analiza cómo medir la intangibilidad de un bien, producto o servicio puesto en el mercado.

Palabras Clave: Calidad, intangibilidad, buenos deseos, adecuación de los servicios, análisis de la calidad del servicio turístico, empatía.

\begin{abstract}
In selling of products or services, marketing function has an important role verifying the needs and expectatives of the consumers. It is analyzed what the customers require when buying a product or service, their preferences, satisfactions and requirements.

Tangiblity of a product goes with the intangibility of the expectatives that must be measured in function of customer's perceptions. It is all about the current article when analyzing how to measure the intangibility of a commodity, product or service in the market.
\end{abstract}

Key Words: Quality of the services, intangible service, goods service, adequate service, quality of the tourism, empathy.

* Doctor en Administración, Magister en Administración y Desarrollo Económico Social, Profesor Principal de la Facultad de Ciencias Administrativas (UNMSM). tres veces «Mérito Científico» de la UNMSM y ponente en los seis últimos Congresos Nacionales de Filosofía. Profesor de postgrado de diversas universidades y centros de enseñanza superior. 


\section{INTRODUCCIÓN}

$\mathrm{Si}$ deseamos medir la mesa del comedor es necesario tomar un centímetro de costurera o un metro de carpintero y efectuar la medición. Si deseáramos medir el ancho de nuestro dormitorio actuaríamos de igual manera. Pero si quisiéramos medir lo intangible ¿cómo lo haríamos? $\mathrm{Si}$ deseamos medir las expectativas de un servicio ¿cómo lo haríamos? ¿Cómo saber si nuestros clientes están satisfechos con nuestro servicio o atención? ¿Cómo saber cuán satisfechos están los turistas que llegaron a nuestro país, con el servicio recibido?

La orientación a la satisfacción del cliente provoca beneficios tangibles y cuantificables en la empresa, e incluso, de ella puede depender la supervivencia de la organización pero ¿cómo saber cual es grado de aceptación que tiene el cliente?

En los últimos años el enfoque «customer satisfaction» se ha convertido en una expresión recurrente en los textos que hablan sobre la relación de la empresa y los integrantes de sus mercados. En la actualidad se afirma que no basta con satisfacer a los clientes, sino que es necesario deleitarlos, e incluso asombrarlos.

Lograr la satisfacción plena del cliente es uno de los objetivos más importante de toda empresa. Los beneficios de alcanzar ese objetivo son los siguientes:

- Un cliente satisfecho vuelve a comprar.

- Un cliente satisfecho comunica a todos sus experiencias positivas con un producto o servicio.

- Un cliente satisfecho deja de lado a la competencia.

- Un turista completamente satisfecho está dispuesto a volver y, sobre todo, a pagar más.

Para que los clientes se formen una opinión positiva, la empresa debe satisfacer sobradamente todas sus necesidades y expectativas.

\section{TIPOS DE CLIENTES QUE UTILIZAN LOS SERVICIOS}

Conocer el tipo de clientes nos permitirá identificar la mejor estrategia a implementar; de otro modo se necesitará un ajuste a la misma.

- Cliente Conservador: persona que teme tomar la decisión de comprar un producto o servicio novedoso debido a que está convencido de que «las cosas anteriores o pasadas fueron mejores».

- Cliente Oportunista: persona que procura obtener una mayor funcionabilidad del producto y servicio, por tanto, tiene la costumbre de presionar al prestador de servicios para obtener mejores ventajas.

- Cliente Tímido: persona que no cuenta con la suficiente confianza y seguridad en sí misma como para tomar una decisión, puesto que teme que los detalles que tiene el producto le ocasionen consecuencias futuras.

- Cliente Pesimista: persona con una actitud negativa, falto de confianza, seguridad y valor. Para él todo es un caos, piensa más en los fracasos que en los triunfos.

- Cliente Escéptico: persona que se muestra incrédula pero tiene una buena actitud de escuchar a su interlocutor.

- Cliente Descontento: es la persona que tiene una actitud de disgusto o desagrado, por un servicio inadecuado o por una experiencia pasada negativa.

- Cliente Hablador: es una persona que habla todo el tiempo y que sus palabras lo estimulan a seguir hablando.

- Cliente Obsesivo: es una persona que es perseverante en exceso por tratar de lograr sus objetivos

- Cliente Amargo: persona poco flexible, reacciona negativamente, es muy sensible. Generalmente está a la defensiva, su conducta se basa en experiencias anteriores negativas.

- Cliente Silencioso: es una persona muy reservada, no expresa fácilmente sus gustos y busca experimentar un clima de confianza.

- Cliente Sarcástico: es una persona burlona, irónica, que tiene una alta estima de sí misma; se cree superior, intachable, difícilmente acepta críticas y cuando las recibe, se ofusca.

- Cliente Sabelotodo: persona autosuficiente, conocedora y experimentada.

- Cliente Ocasional: es una persona que compra eventualmente.

- Cliente Constante: persona que compra regularmente. 
Entonces nuestro servicio tiene que reunir ciertas condiciones para lograr satisfacción de nuestro cliente. Nuestra tarea es lograr clasificar a los turistas, y de acuerdo a ello diseñar estrategias de crecimiento sostenido del sector.

\section{¿QUÉ ES UN SERVICIO?}

A través del tiempo se han desarrollado una serie de definiciones sobre los servicios sin que ninguna obtenga total aceptación en el medio académico por la complejidad del sector servicios:

Definición 1. Servicio (Del lat. servit-um) Acción y efecto de servir || Prestación humana que satisface alguna necesidad social y que no consiste en la producción de bienes materiales || Actividad llevada a cabo por la Administración o, bajo un cierto control y regulación de ésta, por una organización, especializada o no, y destinada a satisfacer necesidades de la colectividad ${ }^{1}$.

Definición 2. Servicios: actividades, beneficios o satisfacciones puestos a la venta o proporcionados en conexión con la venta de bienes ${ }^{2}$.

Definición 3. Los servicios son actividades intangibles e identificables por separado, que proporcionan la satisfacción deseada cuando se venden a los consumidores y/o usuarios industriales y que no están necesariamente vinculadas a la venta de un producto o de otro servicio ${ }^{3}$.

Definición 4. La distinción clave entre productos y servicios radica en el hecho de que los clientes por lo común derivan un valor de los servicios, sin obtener la propiedad permanente de ningún elemento tangible 4 .

Independientemente de las discrepancias de los distintos autores sobre la definición de los servicios, se suelen adoptar algunas de las siguientes posturas sobre el concepto del servicio como objeto de intercambio.

a) Bienes y servicios bajo un mismo concepto globalizador. Bajo este enfoque se considera que no se comercializan ni bienes ni servicios, sino productos que engloban una serie de elementos tangibles e intangibles que configuran un todo.

Esta posición presenta un producto como un todo compuesto por el:
- Producto genérico: recursos básicos.

- Producto esperado: expectativas mínimas requeridas.

- Producto aumentado: beneficios adicionales para aumentar el atractivo del producto.

- Producto potencial: todo lo potencialmente factible para atraer a los clientes.

¿Cuándo decimos que un servicio es exitoso? Cuando el usuario tiene una percepción del servicio recibido que sea superior a la expectativa que tenía previa a la hora de la compra o consumo. ¿Cuándo un servicio es malo? Si la percepción del servicio recibido es inferior a la expectativa que tenía previa al consumo. ¿Cuándo un servicio es indiferente para el cliente? Si el análisis de la percepción es igual a la expectativa para el cliente.

b) Bienes y servicios como conceptos opuestos. Bajo este enfoque se definen los bienes como objetos de intercambios tangibles, y los servicios como objetos de intercambios intangibles.

c) Todos son servicios. Bajo esta postura se considera que no existe diferencia entre el marketing de bienes y el de servicios porque todos son servicios. Esta posición se sustenta en el hecho de que las empresas no venden productos o servicios sino que satisfacen necesidades.

d) Bienes y servicios como un concepto continuo. Bajo este enfoque se considera que los productos tienen componentes tangibles e intangibles en diferentes medidas; los bienes de consumo o industriales se acompañan de servicios para incrementar su valor, de la misma forma como la mayoría de servicios incorporan elementos tangibles que acompañan su comercialización.

\section{CLASIFICACIÓN DE LOS SERVICIOS}

La investigación académica tiene varias propuestas para clasificar los servicios, las cuales ponen de manifiesto la heterogeneidad de actividades que comprenden los servicios.

A continuación se presentan las clasificaciones consideradas más representativas:

a) Naturaleza del servicio. De acuerdo con la naturaleza de la actividad del servicio y los beneficiarios de los servicios, se pueden clasificar en: 
- Procesamiento de Personas. Se refiere a servicios por los cuales las personas pasan a través del proceso y se ejercen acciones tangibles sobre ellos.

- Procesamiento de Estímulo Mental. Se refiere a servicios por los cuales las personas pasan a través del proceso y se ejercen acciones intangibles sobre ellos.

- Procesamiento de Cosas. Se refiere a servicios dirigidos a posesiones físicas en los que el objeto que requiere el procesamiento debe estar presente, pero no es necesario que el cliente esté presente.

- Procesamiento de Información. Se refiere a servicios en los que la participación directa del cliente puede no ser necesaria y se ejercen acciones intangibles sobre activos intangibles.

b) Naturaleza de la demanda y la oferta. Otro criterio de clasificación se refiere a las características de la demanda y a la flexibilidad de la oferta para adaptarse a las fluctuaciones de la demanda.

c) Relación de la empresa con sus clientes. La relación con los clientes puede ser ocasional (un cine) o continua (servicios bancarios), mientras que se pueden establecer relaciones formales (empresa de seguros) o informales (un teatro) con los clientes. La existencia de relaciones formales así como de servicios continuos permite conocer quienes son los clientes y tiene importantes implicancias para la fijación de precios.

d) Grado de personalización del servicio. Hay servicios que tienden a ser desarrollados a la medida como los servicios de salud o la educación individualizada, y otros que tienden a la estandarización como los restaurantes de comida rápida.

e) Método de prestación del servicio. Hay servicios que se prestan a distancia como los servicios financieros, mientras que en otros el cliente debe acudir a la empresa de servicios, como es el caso de una aerolínea. La empresa de servicios también puede desplazarse a donde está el cliente, como es el caso de los servicios de jardinería. La localización del servicio puede ser simple, en un solo local, o múltiple.

\section{CARACTERÍSTICAS DE LOS SERVICIOS}

Los servicios presentan una serie de características a partir de las cuales se define la singularidad del marketing de servicios.

\section{Intangibilidad}

La principal característica de los servicios es su intangibilidad. Los servicios son acciones, satisfacciones, prestaciones y experiencias principalmente; el servicio no se puede ver ni tocar, oler ni degustar.

La intangibilidad genera las siguientes consecuencias:

- Mayor riesgo percibido en la fase previa a la compra, debido a la intangibilidad es difícil para el comprador formarse una idea del servicio antes de adquirirlo.

- Es más difícil exhibir un servicio y lograr su diferenciación, porque sólo es posible aludir a aspectos tangibles como la comodidad, el placer, la tranquilidad, etc., debido a que el servicio en sí mismo no posee atributos que permitan al usuario identificarlo y distinguirlo por características objetivas en las que se base su diferenciación (tamaño, color, calidad de los materiales, diseño, etc.).

- Es más difícil justificar el precio de un servicio, porque los servicios tienen menos características objetivas que los clientes puedan valorar, lo cual genera mayor dificultad al justificar el precio a cobrarse por ellos.

\section{Inseparabilidad}

Los servicios son todo un proceso, por lo tanto ninguna parte de ellos es independiente, se consumen mientras se realizan. La inseparabilidad de los servicios no sólo dificulta el control del nivel de calidad sino que también añade incertidumbre y variabilidad al proceso, ya que incorpora como parte a un nuevo participante: el cliente.

Las principales implicancias de la inseparabilidad son:

- Alta interacción con el personal de contacto, lo cual requiere un alto adiestramiento del personal en términos de su calidad técnica y su calidad funcional.

- Influencia del ambiente físico del lugar donde se presta del servicio. Esto incluye aspectos de decoración, luminosidad, limpieza, el comportamiento de otros clientes. 


\section{Heterogeneidad}

Es imposible la estandarización de servicios puesto que cada unidad de servicio es de algún modo diferente del otro (línea aérea, agencia de viaje, club, restaurante, hotel, etc.).

Se propone la industrialización del servicio, es decir, la sustitución de tecnologías intensivas en mano de obra por tecnologías, tratando de reducir la participación del factor humano.

Las tecnologías planteadas pueden ser:

- Tecnologías Duras, es decir, sustituir el factor humano por tecnología; por ejemplo, los cajeros automáticos o las contestadoras telefónicas.

- Tecnologías Blandas, es decir, sistemas organizados de división del trabajo como es el caso de los restaurantes de comida rápida.

- Tecnologías Híbridas, resultantes de la combinación de equipos con sistemas estándares de trabajo; por ejemplo, los bancos que utilizan cajeros automáticos y servicios personales.

\section{Caducidad}

La caducidad es una característica tanto de los productos como de los servicios, pero en el caso de los servicios es más inmediata. Si no se usa cuando está disponible, la capacidad del servicio se pierde.

Esta situación genera la necesidad de buscar un equilibrio entre la oferta y la demanda dado que no es posible aprovechar los excesos de capacidad en los momentos de baja demanda para generar inventarios de servicio en espera de momentos en los que exista exceso de demanda.

\section{CONCEPTUALIZACIÓN DEL TÉRMINO CALIDAD}

Numerosos son los autores que han abordado el tema de la calidad y generalmente se ha definido como "qué tan adecuado es para el uso»; algunas de estas definiciones son las que se demuestran a continuación:

- Calidad es el resultado de comparación de las expectativas y percepciones (Harrintgton, 1989, Zeithmal, 1991).

- La calidad consiste en satisfacer las demandas del cliente (Espeso y Harvey, 1994).

- Calidad es el conjunto de características que satisfacen las necesidades del consumidor (Juran y Gryna, 1993).
- Edward W. Deming establece que la calidad consiste en exceder las necesidades y expectativas de los clientes a lo largo de la vida del producto.

En todas las definiciones antes mencionadas se resalta de una manera u otra el hecho de que la calidad está en función de la percepción del cliente. La experiencia demuestra que los clientes perciben la calidad de una forma mucho más amplia que el simple hecho de percibir la calidad en el producto adquirido, de ahí que surge la necesidad de que las empresas definan la calidad de la misma manera que lo hacen los clientes.

\section{CALIDAD DEL SERVICIO: DESDE EL PUNTO DE VISTA DEL CONSUMIDOR}

El auge del sector servicios y la creciente competencia ha hecho que la satisfacción del cliente sea el centro de atención de la disciplina del marketing de servicios. Dadas las características de los servicios, la calidad de los mismos surge en los procesos simultáneos de producción, entrega y consumo del servicio, en los cuales el consumidor participa también dentro del proceso.

La calidad del servicio ha sido tradicionalmente concebida -desde el punto de vista del que ofrece el servicio- como la adaptación a las especificaciones establecidas para la prestación. Sin embargo, actualmente la calidad del servicio es concebida desde la perspectiva del cliente. (Ver Gráfico 1)

\section{Gráfico 1}

Relación entre calidad de los servicios y satisfacción del cliente

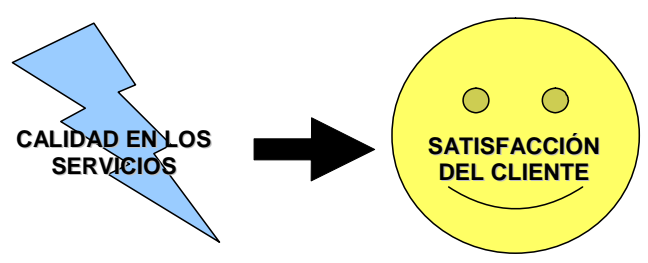

Se plantea que el servicio percibido depende directamente de la llamada calidad técnica (lo que se da, relativo al resultado) y de la funcionalidad (cómo se da, amabilidad, cortesía) que tienen que ver con el desempeño técnico de la prestación del servicio y con el tratamiento dispensado al usuario y en su interacción con los prestadores del servicio, respectivamente. 


\section{SATISFACCIÓN DEL CLIENTE}

Es el nivel del estado de ánimo de una persona que resulta de comparar el rendimiento percibido de un producto o servicio con sus expectativas. ¿Y qué es el Servicio al Cliente? Es la ejecución de todos los medios posibles para dar satisfacción por algo que una persona adquirió.

El servicio es un proceso que implica secuencias, las que se desarrollan previamente en el ámbito interno para luego trasladarlo al exterior de la empresa. Todo proceso de producción o prestación de servicios tiene como objetivo final la satisfacción del cliente.

\section{SENTIMIENTOS QUE INFLUYEN EN EL COMPORTAMIENTO}

- Expectativas. (Del lat. exspectâtum, mirado, visto) Esperanza de realizar o conseguir algo || Posibilidad razonable de que algo suceda ${ }^{5}$. La persona percibe algo que inicialmente es falso o inexistente, y su propia conducta ayuda a que esa creencia se haga realidad. Salvo que se trate de un producto nuevo o desconocido para el consumidor, será el reflejo de otras experiencias anteriores en relación a productos o servicios semejantes, así como las referencias buenas o malas que tenga proveniente de otros consumidores.

- Percepción. (Del lat. perceptiblis) Que se puede comprender o percibir || Que se puede recibir o cobrar ${ }^{6}$. Según James Gibson, la percepción es el proceso por el cual el individuo connota de significado al ambiente. Es un componente de la satisfacción del cliente sobre ciertas dimensiones específicas del servicio como tangibilidad, capacidad de respuesta, seguridad, empatía, fiabilidad.

- Valores. (Del lat. valor, -ôris). Grado de utilidad o aptitud de las cosas para satisfacer las necesidades o proporcionar bienestar o deleite || Cualidad que poseen algunas realidades, consideradas bienes, por lo cual son estimables. Los valores tienen polaridad en cuanto son positivos o negativos, y jerarquía en cuanto son superiores o inferiores ${ }^{7}$.

- Actitudes. (Del lat. actitûdo) Disposición de ánimo manifestada de algún modo ${ }^{8}$.

\section{ESCALAS Y TÉCNICAS DE MEDIDA}

Existen distintas formas de medir o cuantificar las respuestas a determinadas preguntas, principalmente aquellas relacionadas con sentimientos, actitudes, opiniones y creencias. Esto permite sintetizar la información para su uso eficiente y aplicar técnicas por la riqueza de la información.

En la aplicación de las escalas es necesario evaluar tres dimensiones:

- Validez. Analizar si la escala mide aquello que el investigador pretende medir.

- Fiabilidad. Analizar que aquello que se está midiendo se hace de forma consistente.

- Capacidad. Posibilidad de que la escala pueda recoger cambios en el individuo respecto al objeto de medida.

Las principales escalas son las siguientes:

a) Escalas básicas. Son el punto de creación del resto de escalas, desde la nominal a la de ratios, cada una de ellas ofrece mayor precisión en la medición (mayores medidas estadísticas) y en el uso posterior de la información

- Nominal. Se utiliza únicamente para identificar diferentes categorías o alternativas de respuesta. La asignación de valores a las distintas respuestas es arbitraria, los mismos no encierran ningún significado, no indican orden (Ej.: Femenino, Masculino).

- Ordinal. Presenta distintas alternativas de respuesta con diferentes valores que implican rango u orden. El número asociado a los intervalos no tiene ningún significado, sólo una connotación jerárquica (Ej.: primaria, secundaria, universitaria, maestría, doctorado).

- Intervalo. Que presenta distintas alternativas de respuesta con números asociados. Estos números muestran un orden y además, la diferencia entre los valores de la escala es constante y posee significado.

- De Ratios. Tienen las características de las escalas anteriores y además permiten la obtención de ratios coherentes con sus valores. Se conoce perfectamente el punto de origen pudiendo realizarse comparaciones con las distintas respuestas. 
b) Escalas comparativas. Conjunto de escalas en las que las valoraciones se llevan a cabo de forma relativa, atendiendo a un elemento de referencia (conjunto a comparar). Resultan inconvenientes cuando el individuo no tiene conocimiento o experiencia.

- De Comparaciones Pareadas. Se basa en la presentación de los estímulos o elementos a comparar por pares, simplificándose al máximo cada una de las elecciones. Muy utilizada para evaluar productos ya existentes en el mercado.

- De Clasificación. También llamada escala de clasificación por orden de rangos. Se basa en pedir al entrevistado que ordene o clasifique un conjunto de estímulos en función de un atributo.

- De Suma Constante. Se utiliza para medir la importancia relativa que el entrevistado asigna a los estímulos, ya que se le pide que reparta una cantidad de puntos fija (generalmente 100) entre los mismos (el punto de referencia es cero).

- De Clases o Similitudes. Usadas para clasificar a un número elevado de estímulos en un número de subconjuntos o grupos reducidos, atendiendo a la similitud de los mismos. Suele usarse como paso previo a una clasificación ordinal.

- De Protocolos Verbales. Es un tipo de escala en la que se pide la opinión del entrevistado, su posición, frente al estímulo planteado, mostrándose las posibles respuestas en forma de enunciados verbales.

- De Guttman. Es usada como escalograma es menos confiable que la escala de Likert.

c) Escalas no comparativas. No se fundamentan en la comparación entre estímulos o variables, suelen utilizarse para medir valoraciones personales.

- De Clasificación Continua. Diseñadas para medir la opinión de los entrevistados, presentando un elevado número de alternativas de respuesta a través de un continuo. También pueden usarse clasificaciones numéricas.

- Escala Likert. Empleada habitualmente para medir actitudes. Consiste en crear un conjunto de enunciados para que el entrevistado muestre su nivel de acuerdo o desacuerdo.

- De Diferencial Semántico. Evalúa un estímulo en función de diversos atributos, adjetivos o sentencias bipolares, separados por siete categorías de respuesta. Se analizan tanto las puntuaciones totales como los perfiles obtenidos. Puede usarse para analizar y comparar diversos estímulos de forma simultánea.

- Escala Thurstone. Es más laboriosa que la escala de Likert, incluye intervalos de apariencia igual.

- Escala Stapel

d) Escalas estandarizadas. Desarrolladas para estudios específicos (denominaciones propias). Tiene su origen en largas y complejas investigaciones científicas.

- Cetscale (Consumer Etnocentrism). Escala desarrollada para medir el nivel de etnocentrismo de los entrevistados, que consta de 17 proposiciones con las que el entrevistado debe expresar su grado de acuerdo o desacuerdo en uno de los siete niveles posibles.

- Lov (List of Values). Escala usada para la identificación de los estilos de vida, que pretende la medición de distintos valores de los entrevistados.

- Markor. Escala utilizada para medir la orientación al mercado.

- Vals (Values and Life Styles). Escala usada para la identificación de los estilos de vida basada, como la anterior, en un conjunto de valores y actitudes.

- Servperf Se trata de una escala centrada en la medición de la calidad percibida de los servicios, a través de la satisfacción del cliente.

- Servqual. Escala desarrollada con la intención de medir la calidad de los servicios, tanto esperada como percibida. Está formada por 22 declaraciones que se agrupan en cinco dimensiones, cada una de las cuales se identifica con diferentes aspectos relativos a la prestación de servicios. 


\section{MEDICIÓN DE LA CALIDAD DEL SERVICIO}

Nuestros sentidos nos proveen de datos del mundo exterior sin procesar. Estos datos iniciales carecen por completo de significado, por lo que se requiere de un proceso de interpretación para poder encontrar la relación con nosotros.

En las investigaciones de marketing de servicios se han reconocido como instrumentos para medir la calidad del servicio diferentes modelos. Los más estudiados son los de Servqual y el de Servperf. El primero utiliza una escala a partir de las percepciones y expectativas, mientras que el segundo emplea únicamente las percepciones.

El modelo Servqual fue creado por los profesores S. Parasuraman, Valerie Zeithaml y Berry, entre 1985 y 1988 . Éste define la calidad del servicio como la diferencia entre las percepciones reales por parte de los clientes. Es un instrumento de escala múltiple que presenta un alto nivel de fiabilidad y validez, que las empresas pueden utilizar para comprender mejor las expectativas y la percepción que tienen los clientes respecto a un servicio. El modelo incluye dos dimensiones de las expectativas: expectativas deseadas (lo que me gustaría recibir en términos ideales) y expectativas adecuadas (el nivel aceptable de servicio esperado).

Servqual consta de cuatro partes:

- Primera. Se refiere a la medición de las expectativas a través de la calificación que le otorgan los clientes en términos de las empresas excelentes (Measure of Service Superiority) o de las expectativas adecuadas (Measure of Service Adequacy).

- Segunda. Califica la percepción de la calidad del servicio de la empresa analizada. Para cada una de estas dos primeras partes presenta 22 items evaluados en una escala Likert de siete puntos.

- Tercera: Tiene ocho preguntas y hace referencia a la importancia relativa de los cinco criterios.

- Cuarta parte. Se refiere a los datos socios demográficos del encuestado.

Las 22 preguntas referentes a las percepciones y a las expectativas corresponden a las cinco dimensiones de la Calidad:

- Elementos tangibles. Apariencia de las instalaciones físicas, equipos, personal y materiales de comunicación (declaraciones 1 a 4).
- Fiabilidad. Habilidad para ejecutar el servicio prometido de forma fiable y cuidadosa (declaraciones 5 a 9).

- Capacidad de respuesta. Disposición y voluntad de los empleados para ayudar al cliente y proporcionar el servicio (declaraciones 10 a 13).

- Seguridad. Conocimiento y atención mostrados por los empleados (declaraciones 14 a 17).

- Empatía. Habilidades para inspirar credibilidad y confianza (declaraciones 18 a 22).

Para evaluar la calidad un servicio es necesario calcular la diferencia existente entre las puntuaciones de expectativas y percepciones. También se puede estimar la puntuación en cada uno de los cinco criterios de calidad obteniendo un promedio de las puntuaciones individuales, que se obtienen a su vez calificando sus declaraciones para cada una de las dimensiones de calidad. Finalmente se pueden establecer mediciones no ponderadas, es decir, que no tomen en cuenta la importancia relativa de los distintos criterios.

\section{APLICACIONES DEL SERVQUAL}

El modelo planteado tiene múltiples aplicaciones. Por ejemplo, es posible comparar la variación de las expectativas y percepciones a lo largo del tiempo.

Otras de sus aplicaciones son las de:

- Comparar los resultados de una empresa con los resultados de otras empresas competidoras.

- Examinar los segmentos de clientes que poseen diferentes percepciones de calidad analizados según sus características sociodemográficas, psicológicas, la importancia relativa de los criterios en la determinación de sus percepciones de calidad o de las razones de estas percepciones.

- También puede ser aplicado el instrumento para medir las percepciones de los clientes internos sobre la calidad.

La recopilación y análisis de la información se realiza mediante el procesador estadístico SPSS (actualmente versión 14).

Existen otros modelos aplicables al Sector Turismo aparte del Servqual y Servperf, estos son:

- Hotelqual. Este modelo toma como referencia el instrumento Servqual. Es una adapta- 
ción al sector de la hotelería de dicha herramienta.

- Logqual. Plantea que la mejor forma de mensurar la calidad en el sector hotelero es a través del estudio de percepciones de los clientes que hacen contacto con las entidades hoteleras.

- Resortqual. Eficiente en la evaluación a nivel estratégico. Con caracterización del destino y específicamente el polo turístico en cuanto a los elementos que lo componen: tours operadores y cadenas que operan.

\section{CONCLUSIONES}

1. En un mundo globalizado la calidad del servicio es fundamental para competir en cualquier actividad.

2. La medición del servicio es fundamental para todo negocio, para lograr fidelizar a sus clientes.

3. Ahora es factible determinar qué es lo que los clientes valoran. Pero además, hay que tener en cuenta que ese conocimiento debe ser continuo, dinámico, progresivo y adaptable.

4. Las demandas de los clientes son cambiantes; las necesidades, deseos y expectativas de ellos están en continua transformación.

5. Existen varios métodos para medir la calidad del servicio y aplicarla a la actividad turística como el Servqual, Servperf, Hotelqual, Logqual y el Resortqual, entre otros.

6. Felicitar al Ing. F. Becerra quien en el semestre pasado enseño a sus alumnos el Método Servqual en los laboratorios de computo de la Facultad (con las limitaciones de infraestructura del caso).

7. Felicitaciones y reconocimientos a dos profesoras que enaltecen los claustros Sanmarquinos. Ellas son la Dra. Ruth Shady, incansable y batalladora por la defensa de lo auténticamente nuestro como son los restos arqueológicos de Caral, y la Lic. Elizabeth Canales, alcaldesa de Lunahuaná, donde cinco universidades han desplazado sus aulas para la enseñanza. Un bonito ejemplo para los alumnos de la Escuela de Turismo de la Facultad.

\section{NOTAS}

1 Microsoft ${ }^{\circledR}$ Encarta ${ }^{\circledR}$ 2006. C $1993-2005$ Microsoft Corporation.

2 American Marketing Association

3 Stanton

4 Lovelock

5 Microsoft $\left(\right.$ Encarta ${ }^{\circledR}$ 2006. (C) 1993-2005 Microsoft Corporation.

6 Op. cit.

7 Op. cit.

8 Op. cit.

\section{BIBLIOGRAFÍA}

Becerra Grande A.; Falces Delgado C.; Sierra Dial B. Hotelqual: una Escala para medir Calidad Percibida en Servicios de Alojamiento.

Berry l. Marketing en las Empresas de Servicios; compita mediante la Calidad. Colombia. Editorial Norma.

Berry l. L. y Parasuraman A. (1991). Marketing de servicios, la Calidad como Meta. Nueva York, Free Press.

Cronin J. y Taylor S. (1994). Servperf vs. Servqual, reconciling performance based and perceptions minus expectations measurement of Service Quality. Journal of Marketing, Vol. 58.

Crosby Lehtinen J. (1983). Compañía de Servicios orientado al Cliente. Finlandia, Espoo.

Deming E. (1990) Four Day Deming Seminar. Jan 30feb 2 1990, Washington, D.C.

Juran J. (1994). Manual de Control de la Calidad. Cuarta Edición.

Kotler P. (1991). Dirección de Marketing. Análisis, Planificación y Control. Séptima Edición. España. Ed Prentice.

Parasuraman A.; Zeithaml V.A. y Berry l. (1994). Alternative Scales for measuring Service Quality: a comparative assessment based on psychometric and diagnostic criteria. 